\title{
Dinâmica da recuperação ambiental de pilhas de estéril em mineração de calcário por regeneração natural
}

\author{
Environmental recovery dynamics of waste dumps \\ in calcary mining by natural regeneration \\ Marcelle Teodoro Lima** (), Kelly Cristina Tonello' ${ }^{\circledR}$, Eliana Cardoso Leite ${ }^{1}$, \\ Fernando Silveira Franco ${ }^{1}\left(\mathbb{0}\right.$, Carina Júlia Pensa Corrêa' ${ }^{1}$ ()
}

口-

\begin{abstract}
RESUMO
A presente pesquisa teve como objetivo avaliar a dinâmica da recuperação ambiental de pilhas de estéril de mineração de calcário por meio da análise da composição e estrutura fitossociológica da regeneração natural. O estudo foi conduzido em um empreendimento minerário localizado em Salto de Pirapora (SP), Brasil, com pilhas de estéril abandonadas desde 1994 e 2013, e um fragmento florestal nativo, totalizando três tratamentos. Em cada área, foram alocadas 12 parcelas de $10 \times 10 \mathrm{~m}$, onde todos os indivíduos arbustivoarbóreos com altura acima de 1,30 m foram amostrados, identificados e avaliados quanto à composição e à estrutura fitossociológica. Foi constatada a ocorrência da regeneração natural nas pilhas de estéril com o aumento de indivíduos e espécies ao longo do tempo. Os resultados indicam que a vegetação presente nessas pilhas está em transição de floresta pioneira para um estágio sucessional mais avançado. Contudo, a Leucaena leucocephala estabeleceu-se mais expressivamente que as demais, inibindo o desenvolvimento de outras espécies. Por fim, sugerem-se ações de manejo para que a sucessão ecológica das pilhas de estéril não seja comprometida.
\end{abstract}

Palavras-chave: gestão ambiental; resíduos sólidos; áreas degradadas.

\begin{abstract}
The present research had as objective to evaluate the dynamics of the environmental recovery of waste dumps in calcary mining by analyzing the composition and phytosociological structure of natural regeneration. The study was carried out in a waste dumo located in Salto de Pirapora, São Paulo, Brazil, with sterile piles abandoned since 1994 and 2013 and a native forest fragment, totaling three treatments. Twelve plots of $10 \times 10 \mathrm{~m}$ were allocated to each area, where all shrub-tree individuals with height above $1.30 \mathrm{~m}$ were sampled, identified and evaluated for their composition and phytosociological structure. The occurrence of natural regeneration in the sterile cells was observed with the increased number of individuals and species over time. The results indicate that the vegetation present in the waste dumps is in transition from pioneer forest to a more advanced successional stage. However, Leucaena leucocephala has established itself more expressively than other species, inhibiting the development of other species. Finally, management actions are suggested so that the ecological succession of the waste dumps is not compromised.
\end{abstract}

Keywords: environmental management; solid waste; degraded areas.

\section{INTRODUÇÃO}

Os bens minerais são essenciais para o desenvolvimento da humanidade. O calcário, por exemplo, é usado como matéria-prima na construção civil, na fabricação de cal e cimento, e tem grande importância na agricultura, como corretivo para solos ácidos. Além disso, é empregado nas indústrias de papel, plástico, química, siderúrgica, de vidro e refratários.

A maior parte das minas de calcário no mundo é lavrada a céu aberto, nas chamadas pedreiras, e as principais etapas da lavra de calcário nessas condições incluem remoção do capeamento, perfuração, desmonte por explosivos e transporte até a usina de processamento (SAMPAIO; ALMEIDA, 2005). Para obtenção do mineral, resíduos sólidos são gerados, tais como estéreis e rejeitos.

Estéreis são os materiais escavados gerados pelas atividades de extração ou lavra no decapeamento da mina, não têm valor econômico e ficam geralmente dispostos em pilhas. Já os rejeitos são os resíduos resultantes dos processos de beneficiamento a que são submetidas as substâncias minerais (SILVA; VIANA; CAVALCANTI, 2011). A extração por recursos minerais ocasiona também impactos significativos 
ao ambiente, seja quanto à exploração de áreas naturais seja quanto à geração de resíduos.

A recuperação de áreas degradadas passou a ser parte da mineração com o Decreto no 97.632/89 (BRASIL, 1989), que dispõe sobre Plano de Recuperação de Área Degradada (PRAD) pela mineração. Esse instrumento jurídico e político determina que o responsável pela atividade minerária recupere a área explorada, a fim de que esta possa voltar a desempenhar um papel relevante na sociedade.

Existem técnicas e modelos para recuperar áreas degradadas, e a sua escolha dependerá dos níveis de degradação encontrados, das características e do uso futuro da área a ser recuperada. Uma das técnicas utilizadas através da restauração ecológica é a regeneração natural, que consiste no processo pelo qual espécies florestais nativas se estabelecem em áreas alteradas ou degradadas a serem recuperadas ou em recuperação, sem que esse processo tenha ocorrido deliberadamente por meio de intervenção humana (BRASIL, 2017).

Em áreas onde a ocorrência de perturbações é constante e intensa, o processo de restauração pode se tornar lento ou mesmo ser inibido (MAGNAGO et al., 2012). Isso pode reduzir o avanço do processo de sucessão ecológica e, consequentemente, comprometer o aumento da complexidade estrutural da vegetação e perpetuação das espécies vegetais (GANDOLFI; LEITÃO FILHO; BEZERRA, 1995; MIRANDA NETO et al., 2012). Outro aspecto importante de mudanças sucessionais está relacionado à invasão de espécies exóticas, que representa notável ameaça à biodiversidade (PRACH; WALKER, 2011).

Entender como se dá o processo de sucessão ecológica em áreas perturbadas é importante para aperfeiçoar técnicas de recuperação da cobertura florestal. Compreender a dinâmica da vegetação possibilitará ampliar teorias de sucessão e os esforços na restauração de florestas tropicais (HOLL, 2002). Para Sartori (2001), a necessidade de se conhecer a composição e estrutura da vegetação natural em condições de perturbação e regeneração subsequente é ponto primordial para as decisões práticas que têm por objetivo a restauração ecológica do ecossistema. Segundo McIntire e Fajardo (2014), estudar a sucessão ecológica tem como objetivo identificar quais são as interações negativas e positivas no processo e como potencializá-las para aumentar a biodiversidade.

Diante da problemática da recuperação ambiental de áreas degradadas de mineração, este estudo teve como objetivo avaliar a dinâmica da recuperação ambiental de pilhas de estéril de mineração de calcário, em diferentes idades, por meio da composição e estrutura fitossociológica da vegetação. Com os resultados encontrados, espera-se fornecer subsídios à recuperação de áreas degradadas pela mineração.

\section{METODOLOGIA}

A pesquisa foi conduzida em uma empresa mineradora de calcário para insumos agrícolas e construção civil, localizada em Salto de Pirapora, no estado de São Paulo, Brasil. Com uma extensão de 26.657,88 hectares, a mineradora encontra-se entre as longitudes $47^{\circ} 31^{\prime} 52.12^{\prime \prime} \mathrm{e}$

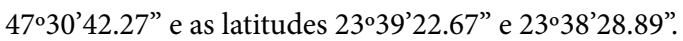

De acordo com o Instituto Brasileiro de Geografia e Estatística (IBGE, 2012), a área de estudo está inserida em um ecótono, com formação vegetal originalmente composta de floresta estacional semidecidual e zonas de contato com cerrado.

O clima da região, segundo a classificação de Koeppen, é do tipo Cwa, tropical de altitude com chuvas no verão e seca no inverno, com temperatura média do mês mais quente superior a $22^{\circ} \mathrm{C}$ (CEPAGRI; UNICAMP, 2016).

Para avaliação da regeneração natural, foi realizado o levantamento da composição e estrutura fitossociológica em pilhas de estéril abandonadas desde 2013 (área I) e 1994 (área II) e em uma área de referência (AR) com fragmento florestal nativo adjacente a uma cava de mineração.

A área de estudo correspondeu a $3.600 \mathrm{~m}^{2}$, onde foram determinadas 12 parcelas de $10 \times 10 \mathrm{~m}$ em cada tratamento (área 1 , área $2 \mathrm{e}$ $\mathrm{AR})$, com espaçamento de $10 \mathrm{~m}$ entre si. Nessas áreas, todos os indivíduos lenhosos vivos (árvores, arbustos e subarbustos), com altura $\geq 1,30 \mathrm{~m}$ do solo, tiveram o diâmetro à altura do peito (DAP) registrado e foram identificados e marcados com plaquetas de alumínio. O reconhecimento das espécies foi realizado in situ, quando possível, ou partes vegetativas e/ou reprodutivas das plantas não reconhecidas no local foram coletadas para identificação por meio de literatura especializada e consulta a herbários. As famílias foram nomeadas com base no Angiosperm Phylogeny Group III (APG III, 2009; SOUZA; LORENZI, 2008). Grafia dos nomes e sinonímias das espécies foram confirmados de acordo com a Flora do Brasil 2020 (2016) e classificadas quanto ao tipo (nativa ou exótica) e à categoria sucessional (pioneira e não pioneira). As coletas ocorreram no período de julho de 2015 a junho de 2016.

Com o auxílio do software Fitopac 2.1 (SHEPHERD, 2009), obtiveram-se as variáveis fitossociológicas da estrutura horizontal da vegetação: frequências absoluta (FA) e relativa (FR), densidades absoluta (DA) e relativa (DR), dominâncias absoluta (DoA) e relativa (DoR) e índices de valores de importância (IVI) e de cobertura (IVC) (MUELLER-DOMBOIS; ELLENBERG, 1974) (Tabela 1). A diversidade florística foi estimada por meio do índice de diversidade de Shannon-Wiener (H') (MAGURRAN, 1988), e a similaridade florística (matriz de presença-ausência) entre os ambientes foi analisada pelo índice de Sørensen, sendo que, para melhor entendimento, os resultados foram apresentados em porcentagem (WOLDA, 1981).

Para avaliar o possível impacto de plantas exóticas sobre as nativas, foi medido o Índice de Impacto Ambiental de Exóticas (IIAE), por meio do cálculo dos coeficientes de impacto ambiental (REASER et al., 2007). 


\section{RESULTADOS E DISCUSSÃO}

$\mathrm{Na}$ área total amostrada, foram inventariados 1.622 indivíduos, distribuídos em 50 espécies e 27 famílias (Tabelas 1, 2 e 3).

$\mathrm{Na}$ área I, foram encontrados 309 indivíduos, 11 espécies e 6 famílias. As espécies mais representativas foram a Leucaena leucocephala
(179) e a Trema micranta (59), correspondendo a 77\% dos indivíduos amostrados e, por consequência, suas respectivas famílias: Fabaceae (188) e Cannabaceae (59).

Na área II, houve um incremento nos números de indivíduos (700), espécies (18) e famílias (10), o que indica um avanço no processo

Tabela 1 - Relação das espécies amostradas nas pilhas de estéril em mineração de calcário abandonada desde 2013 (área I), com os respectivos parâmetros fitossociológicos em ordem decrescente de maior índice de valor de importância.

\begin{tabular}{|c|c|c|c|c|c|c|c|c|c|}
\hline Espécie & Familia & $\mathrm{N}$ & $\mathrm{DR}$ & FR & DoR & IVI & IVC & CS & OR \\
\hline Leucaena leucocephala (Lam.) de Wit & Fabaceae & 179 & 57,90 & 22,90 & 53,90 & 135,00 & 112,000 & $P$ & E \\
\hline Trema micrantha (L.) Blume & Cannabaceae & 59 & 19,10 & 14,60 & 36,70 & 70,40 & 55,800 & $\mathrm{P}$ & N \\
\hline Tecoma stans (L.) Kunth & Bignoniaceae & 16 & 5,18 & 10,40 & 1,97 & 17,60 & 7,150 & $P$ & $E$ \\
\hline Baccharis uncinella DC. & Asteraceae & 18 & 5,83 & 8,33 & 0,91 & 15,10 & 6,740 & $\mathrm{P}$ & N \\
\hline Vernonanthura tweediana (Baker) H. Rob. & Asteraceae & 15 & 4,85 & 8,33 & 1,60 & 14,80 & 6,460 & $\mathrm{P}$ & N \\
\hline Baccharis oblongifolia (Ruiz \& Pav.) Pers. & Asteraceae & 5 & 1,62 & 8,33 & 0,78 & 10,70 & 2,390 & $P$ & $\mathrm{~N}$ \\
\hline Mikania sericea Hook. \& Arn. & Asteraceae & 4 & 1,29 & 8,33 & 0,34 & 9,97 & 1,630 & $\mathrm{P}$ & N \\
\hline Sesbania punicea (Cav.) Benth. & Fabaceae & 4 & 1,29 & 6,25 & 1,42 & 8,97 & 2,720 & $\mathrm{P}$ & N \\
\hline $\begin{array}{l}\text { Senegalia bonariensis (Gillies ex Hook. \& Arn.) Seigler } \\
\text { \& Ebinger }\end{array}$ & Fabaceae & 5 & 1,62 & 6,25 & 0,80 & 8,66 & 2,410 & $\mathrm{P}$ & N \\
\hline Piper dilatatum Rich. & Piperaceae & 2 & 0,65 & 4,17 & 0,24 & 5,05 & 0,88 & $\mathrm{P}$ & N \\
\hline Solanum viarum Dunal & Solanaceae & 2 & 0,65 & 2,08 & 1,35 & 4,08 & 2 & $\mathrm{P}$ & $\mathrm{N}$ \\
\hline
\end{tabular}

N: número de indivíduos; DR: densidade relativa; FR: frequência relativa; DoR: dominância relativa; IVI: índice de valor de importância; IVC: índice de valor de cobertura; CS: categoria sucessional (P: pioneira); OR: origem (E: exótica, N: nativa).

Tabela 2 - Relação das espécies amostradas nas pilhas de estéril em mineração de calcário abandonada desde 1994 (área II), com os respectivos parâmetros fitossociológicos em ordem decrescente de maior índice de valor de importância.

\begin{tabular}{|c|c|c|c|c|c|c|c|c|c|}
\hline Espécie & Familia & $\mathrm{N}$ & DR & FR & DoR & IVI & IVC & CS & OR \\
\hline Leucaena leucocephala (Lam.) de Wit & Fabaceae & 576 & 82,0 & 15,30 & 82,0 & 179,00 & 164,0 & $\mathrm{P}$ & E \\
\hline Aloysia virgata (Ruiz \& Pav.) Juss. & Verbanaceae & 21 & 3,0 & 11,90 & 0,6 & 15,40 & 3,6 & $\mathrm{P}$ & $N$ \\
\hline Lithraea molleoides (Vell.) Engl. & Anacardiaceae & 10 & 1,4 & 11,90 & 1,2 & 14,50 & 2,6 & $\mathrm{P}$ & $N$ \\
\hline Tecoma stans (L.) Kunth & Bignoniaceae & 12 & 1,7 & 10,20 & 0,3 & 12,20 & 2,1 & $\mathrm{P}$ & E \\
\hline Dalbergia frutescens (Vell.) Britton & Fabaceae & 8 & 1,1 & 3,39 & 7,2 & 11,80 & 8,4 & $\mathrm{P}$ & $\mathrm{N}$ \\
\hline Piper dilatatum Rich. & Piperaceae & 24 & 3,4 & 6,78 & 0,7 & 10,90 & 4,1 & $P$ & $N$ \\
\hline Psidium guajava L. & Myrtaceae & 10 & 1,4 & 8,47 & 0,3 & 10,20 & 1,7 & $\mathrm{P}$ & $N$ \\
\hline Baccharis oblongifolia (Ruiz \& Pav.) Pers. & Asteraceae & 16 & 2,3 & 6,78 & 0,4 & 9,50 & 2,7 & $\mathrm{P}$ & N \\
\hline Moquiniastrum polymorphum (Less.) G. Sancho & Asteraceae & 6 & 0,9 & 5,08 & 2,6 & 8,52 & 3,4 & $P$ & $\mathrm{~N}$ \\
\hline $\begin{array}{l}\text { Senegalia bonariensis (Gillies ex Hook. \& Arn.) Seigler } \\
\text { \& Ebinger }\end{array}$ & Fabaceae & 3 & 0,4 & 3,39 & 2,9 & 6,70 & 3,3 & $P$ & $N$ \\
\hline Vernonanthura phosphorica (Vell.) H.Rob & Asteraceae & 4 & 0,6 & 3,39 & 0,1 & 4,02 & 0,6 & P & $N$ \\
\hline Solanum viarum Dunal. & Solanaceae & 2 & 0,3 & 3,39 & 0,1 & 3,76 & 0,4 & $\mathrm{P}$ & N \\
\hline Trema micrantha (L.) Blume & Cannabaceae & 1 & 0,1 & 1,69 & 1,3 & 3,14 & 1,5 & $\mathrm{P}$ & $\mathrm{N}$ \\
\hline $\begin{array}{l}\text { Dahlstedtia muehlbergiana (Hassl.) M.J.Silva \& A.M.G. } \\
\text { Azevedo }\end{array}$ & Fabaceae & 1 & 0,1 & 1,69 & 0,4 & 2,26 & 0,6 & NP & $N$ \\
\hline Chromolaena odorata (L.) R.M.King \& H.Rob. & Asteraceae & 2 & 0,3 & 1,69 & O & 1,99 & 0,3 & $P$ & N \\
\hline Nectandra megapotamica (Spreng.) Mez & Lauraceae & 2 & 0,3 & 1,69 & O & 1,99 & 0,3 & NP & $\mathrm{N}$ \\
\hline Mikania sericea Hook. \& Arn. & Asteraceae & 1 & 0,1 & 1,69 & O & 1,86 & 0,2 & $\mathrm{P}$ & N \\
\hline Baccharis punctulata DC. & Asteraceae & 1 & 0,1 & 1,69 & 0 & 1,84 & 0,2 & $\mathrm{P}$ & N \\
\hline
\end{tabular}

N: número de indivíduos; DR: densidade relativa; FR: frequência relativa; DoR: dominância relativa; IVI: índice de valor de importância; IVC: índice de valor de cobertura; CS: categoria sucessional (P: pioneira, NP: não pioneira); OR: origem (E: exótica, N: nativa). 
de desenvolvimento da cobertura florestal. Assim como na área I, a Leucaena leucocephala foi a espécie de maior ocorrência (576), seguida da Piper dilatatum (24) e da Allysia virgata (21), o que totalizou 89\%, aproximadamente. Quanto às famílias, as mais representativas foram Fabaceae (588), Asteraceae (30) e Piperaceae (24).
$\mathrm{Na}$ AR, foram amostrados 613 indivíduos, 34 espécies e 22 famílias. As espécies de maior ocorrência foram Piper amalago (143), Nectandra megapotamica (126), Machaerium sapitatum (75), Eriobothrya japonica (54) e Cupania vernalis (33), perfazendo aproximadamente $70 \%$ do total. Os indivíduos estão distribuídos em Piperaceae (149), Lauraceae

Tabela 3 - Relação das espécies amostradas na área de referência em ordem decrescente de maior índice de valor de importância.

\begin{tabular}{|c|c|c|c|c|c|c|c|c|c|}
\hline Espécie & Familia & $\mathrm{N}$ & DR & $\mathrm{FR}$ & DoR & IVI & IVC & CS & OR \\
\hline Machaerium stipitatum Vogel & Fabaceae & 75 & 12,20 & 7,59 & 68,40 & 88,20 & 80,60 & $\mathrm{P}$ & N \\
\hline Nectandra megapotamica (Spreng.) Mez & Lauraceae & 126 & 20,60 & 7,59 & 10,80 & 38,90 & 31,30 & NP & N \\
\hline Piper amalago L. & Piperaceae & 143 & 23,30 & 7,59 & 0,91 & 31,80 & 24,20 & $\mathrm{P}$ & $\mathrm{N}$ \\
\hline Cupania vernalis Cambess. & Sapindaceae & 33 & 5,38 & 6,96 & 3,61 & 16,00 & 8,99 & NP & N \\
\hline Eriobothrya japonica (Thunb.) Lindl. & Rosaceae & 54 & 8,81 & 5,70 & 0,71 & 15,20 & 9,52 & $\mathrm{P}$ & $E$ \\
\hline Trichilia elegans A. Juss. & Meliaceae & 25 & 4,08 & 6,33 & 2,28 & 12,70 & 6,36 & NP & $\mathrm{N}$ \\
\hline Ceiba speciosa (St.-Hill.) & Malvaceae & 4 & 0,65 & 2,53 & 4,32 & 7,50 & 4,97 & NP & $\mathrm{N}$ \\
\hline Matayba elaeagnoides Radlk. & Sapindaceae & 13 & 2,12 & 4,43 & 0,53 & 7,08 & 2,65 & NP & $\mathrm{N}$ \\
\hline Myrsine parvula (Mez) Otegui & Primulaceae & 14 & 2,28 & 4,43 & 0,29 & 7,00 & 2,57 & $\mathrm{P}$ & $\mathrm{N}$ \\
\hline $\begin{array}{l}\text { Allophylus edulis (A.St.-Hil., Cambess. \& A. Juss.) } \\
\text { Radlk. }\end{array}$ & Sapindaceae & 12 & 1,96 & 2,53 & 1,33 & 5,82 & 3,28 & P & $N$ \\
\hline Dalbergia frutescens (Vell.) Britton & Fabaceae & 10 & 1,63 & 2,53 & 1,30 & 5,47 & 2,94 & NP & $\mathrm{N}$ \\
\hline Guarea macrophylla Vahl & Meliaceae & 11 & 1,79 & 3,16 & 0,34 & 5,30 & 2,13 & NP & N \\
\hline Styrax leprosus Hook. \& Arn. & Styracaceae & 9 & 1,47 & 2,53 & 1,22 & 5,22 & 2,69 & $\mathrm{P}$ & N \\
\hline Casearia sylvestris Sw. & Salicaceae & 7 & 1,14 & 3,16 & 0,81 & 5,12 & 1,96 & $\mathrm{P}$ & $\mathrm{N}$ \\
\hline Piper dilatatum Rich. & Piperaceae & 6 & 0,98 & 3,80 & 0,02 & 4,79 & 0,99 & $P$ & N \\
\hline Zanthoxylum rhoifolium Lam. & Rutaceae & 6 & 0,98 & 3,16 & 0,42 & 4,56 & 1,40 & $\mathrm{P}$ & N \\
\hline Erythroxylum pelleterianum A. St.-Hil. & Erythroxylaceae & 8 & 1,31 & 3,16 & 0,06 & 4,53 & 1,37 & NP & N \\
\hline Xylopia brasiliensis Spreng. & Annonaceae & 6 & 0,98 & 3,16 & 0,10 & 4,24 & 1,08 & NP & $\mathrm{N}$ \\
\hline $\begin{array}{l}\text { Chrysophyllum gonocarpum (Mart. \& } \\
\text { Eichler ex Miq.) Engl. }\end{array}$ & Sapotaceae & 5 & 0,82 & 2,53 & 0,26 & 3,60 & 1,07 & $N P$ & $N$ \\
\hline Cedrela fissilis Vell. & Meliaceae & 9 & 1,47 & 1,90 & 0,07 & 3,44 & 1,54 & NP & N \\
\hline Piptadenia gonoacantha (Mart.) J.F.Macbr. & Fabaceae & 5 & 0,82 & 1,90 & 0,66 & 3,37 & 1,47 & NP & N \\
\hline Solanaceae 1 & Solanaceae & 3 & 0,49 & 1,90 & 0,70 & 3,09 & 1,19 & - & - \\
\hline Citrus limon (L.) Burm. f. & Rutaceae & 5 & 0,82 & 1,90 & 0,03 & 2,74 & 0,84 & $\mathrm{P}$ & E \\
\hline Solanaceae 2 & Solanaceae & 4 & 0,65 & 1,90 & 0,02 & 2,57 & 0,68 & - & - \\
\hline Dahlstedtia muehlbergiana & Fabaceae & 6 & 0,98 & 1,27 & 0,10 & 2,34 & 1,08 & $\mathrm{NP}$ & $\mathrm{N}$ \\
\hline Croton floribundus Spreng & Euphorbiaceae & 3 & 0,49 & 1,27 & 0,02 & 1,77 & 0,51 & $\mathrm{P}$ & N \\
\hline Lithraea molleoides (Vell.) Engl. & Anacardiaceae & 1 & 0,16 & 0,63 & 0,63 & 1,42 & 0,79 & NP & $\mathrm{N}$ \\
\hline Mangifera sp & Anacardiaceae & 2 & 0,33 & 0,63 & 0,05 & 1,01 & 0,38 & NP & $E$ \\
\hline Cariniana estrellensis (Raddi) Kuntze & Lecythidaceae & 2 & 0,33 & 0,63 & 0,02 & 0,98 & 0,35 & NP & $\mathrm{N}$ \\
\hline Guapira opposita (Vell.) Reitz & Nyctaginaceae & 2 & 0,33 & 0,63 & 0,01 & 0,96 & 0,33 & NP & $\mathrm{N}$ \\
\hline Campomanesia cf xanthocarpa O. Berg & Myrtaceae & 1 & 0,16 & 0,63 & 0,05 & 0,85 & 0,21 & NP & $\mathrm{N}$ \\
\hline Indeterminado & Indeterminado & 1 & 0,16 & 0,63 & 0,02 & 0,81 & 0,18 & - & - \\
\hline Araliaceae & Araliaceae & 1 & 0,16 & 0,63 & 0,01 & 0,8 & 0,17 & - & - \\
\hline Garcinia sp. & Clusiaceae & 1 & 0,16 & 0,63 & 0 & 0,8 & 0,17 & NP & E \\
\hline
\end{tabular}

N: número de indivíduos; DR: densidade relativa; FR: frequência relativa; DoR: dominância relativa; IVI: índice de valor de importância; IVC: índice de valor de cobertura; CS: categoria sucessional (P: pioneira, NP: não pioneira); OR: origem (E: exótica, N: nativa). 
(126), Fabaceae (96) e Sapindaceae (58). No caso da AR, houve um indivíduo indeterminado.

Um estudo realizado por Cielo-Filho e Souza (2016) avaliou a regeneração natural de uma área da mata atlântica após o corte raso de uma plantação de Cupressus lusitânica, onde os resultados mostraram um nível relativamente elevado de eficácia da regeneração natural em recuperar a riqueza de espécies, diversidade, síndromes de dispersão e composição.

Em nenhuma das áreas, houve plantio ou enriquecimento com espécies florestais para recuperar aquelas degradadas pela deposição de estéril. Tanto na área I como na II, a espécie Leucaena leucocephala obteve o maior IVC e IVI, diferentemente da AR, onde esses índices foram maiores para Machaerium stipitatum. Contudo, ambas as espécies pertencem à família Fabaceae, reconhecida por sua comprovada capacidade de fixação de nitrogênio.

Outra espécie de destaque foi a Tecoma stans, cujo IVI ocupou a $3^{\circ}$ e a $4^{\circ}$ posições nas áreas I e II, respectivamente. Sabe-se que essa planta está inclusa na Lista de Espécies Exóticas Invasoras do Paraná, e a Portaria $\mathrm{n}^{\circ} 125$, de 7 de agosto de 2009, estabelece que ela não deve ser cultivada ou criada, ficando seu uso, em qualquer uma das formas, não permitido (IAP, 2009). Estudos realizados por Passini (1996) descrevem que a Tecoma stans comportou-se como planta invasora de pastos degradados do estado do Paraná. A população dessa espécie forma um dossel denso, sob o qual as forrageiras não se desenvolvem.

A AR, embora ainda seja uma floresta jovem em desenvolvimento sucessional, apresentou estrutura e composição mais complexa que nas áreas I e II. Além da presença da família Piperaceae, o avanço da sucessão pode ser verificado pela presença da Lauraceae como a mais representativa. Para Tabarelli (1994), essa família é indicativa da transição da floresta pioneira para um estágio sucessional mais avançado no domínio da floresta atlântica. Na área II, a família Piperaceae é a segunda maior riqueza, sendo indicativa de que a vegetação presente nas pilhas de estéril está em avanço de sucessão ecológica. No sub-bosque, elas tendem a ocorrer mais frequentemente em áreas menos sombreadas.

$\mathrm{Na}$ distribuição das espécies em grupo ecológico, as pioneiras predominaram em número de indivíduos e espécies nas áreas I (100\%) e II $(88,8 \%)$. Esse grupo é considerado chave para o processo de recuperação. De acordo com Rodrigues, Monteiro e Cullen (2010), as pioneiras são responsáveis pelo impulso inicial, pelo rápido recobrimento do solo e pela criação de condições para o estabelecimento de outras espécies. No entanto, de acordo com Brancalion, Gandolfi e Rodrigues (2009), uma densidade elevada de espécies pioneiras pode comprometer os processos ecológicos futuros da área em restauração, principalmente quando essa área estiver em local distante de fontes de propágulos (fragmentos florestais) ou desprovida de sementes armazenadas no solo, o que inviabiliza o processo de sucessão florestal. Contudo, como existem remanescentes florestais em estágio sucessional mais avançado nas proximidades dos depósitos de estéril, o enriquecimento com espécies finais de sucessão deverá ocorrer de forma natural.

Foi observado, na área II, o início do estabelecimento de espécies não pioneiras, as quais corresponderam a $11,1 \%$ dos indivíduos amostrados, distribuídos em 18 espécies. Verificou-se ainda que o sombreamento proporcionado pelo dossel já está possibilitando a regeneração de espécies de estágios mais avançados, como Nectandra megapotamica e Dahlstedtia muehlbergiana. Espera-se, com o passar do tempo, que as espécies pioneiras sejam substituídas por aquelas de sucessão mais avançadas.

Os índices de diversidade de Shannon ( $\left.\mathrm{H}^{\prime}\right)$ obtidos nas áreas I e II e na AR foram, respectivamente, de 1,40; 0,89; e 2,57 nats.indivíduo $^{-1}$. De maneira geral, os valores de $\mathrm{H}^{\prime}$ encontrados nas áreas foram menores se comparados com estudos sobre vegetação no estado de São Paulo, que variaram de 3,04 a 4,01 nats.indivíduo-1 (DISLICH; CERSÓSIMO; MANTOVANI, 2001; COELHO, 2013; CORRÊA et al., 2014; CARDOSO-LEITE; RODRIGUES, 2008). Percebe-se que as áreas I e II representaram H' menores quando comparados com AR.

Cabe salientar que uma área restaurada não necessariamente ficará igual a uma área com vegetação conservada e que esse processo de restauração pode levar muito tempo para ocorrer. Entretanto, a comparação torna-se mais confiável com áreas que apresentam histórico de degradação semelhante (MARTINS; COUTINHO; MARANGON, 2002), ainda que recuperadas de maneira antrópica (restauração ativa). Em áreas de mineração de bauxita recuperadas ativamente há 10 anos na Amazônia, o H’ variou de 0,65 a 0,85 nats.indivíduo-1 (PARROTTA; KNOWLES; WUNDERLE JR., 1997). Foram encontrados resultados semelhantes em área de mineração de ferro em Mariana (MG), recuperada de maneira ativa, onde o $\mathrm{H}^{\prime}$ variou de 0,91 a 1,17 nats.indivíduo $^{-1}$, de acordo com a idade de restauração de cada sítio ( 7 a 17 anos) (ÂNGELO et al., 2002).

Ao considerar que, nas pilhas de rejeito, não houve nenhuma intervenção humana para recuperação das áreas, os índices de diversidade obtidos neste trabalho apresentaram valores semelhantes. Nota-se ainda que o H' da área I foi maior que o da II, apesar de ser a pilha de estéril mais velha. Esse fato pode ser justificado pelo critério de mensuração de DAP da vegetação, por meio do qual foram mensurados todos os indivíduos lenhosos com altura $\geq 1,30 \mathrm{~m}$ do solo, o que resultou em uma grande quantidade de arbustos e elevou o H' da área I.

O índice de Sorensen entre as áreas I e II foi de 62,0\%; entre a I e a AR, 6,6\%; e entre a II e a AR, 19,2\%. Os resultados indicam que a maior semelhança ocorreu entre as áreas I e II. Ao comparar as pilhas de estéril com a AR, os valores encontrados foram baixos, o que representa baixa 
similaridade florística entre as áreas. Entretanto, a maior similaridade foi observada na comparação entre a área II e a AR, o que sugere que, com o avançar do tempo, as pilhas de estéril tendem a se assemelhar à $\mathrm{AR}$, ainda que lentamente.

A baixa riqueza específica nas pilhas de estéril é ratificada também pelo IIAE, cujos valores obtidos foram 0,014 e 0,277 para as áreas I e II, respectivamente. Sob o contexto estrutural, observou-se que a Leucaena leucocephala representou o maior valor de importância, especialmente devido à sua alta dominância e distribuição homogênea. Nas pilhas de estéril, observou-se também um menor número de indivíduos representativos das espécies nativas quando comparadas com AR. Segundo Reaser et al. (2007), o IIAE varia de -1 a 1 e, quando o valor analisado é -1 , implica que a área não conta com plantas nativas. Quando o valor analisado é 1, a área não tem plantas exóticas. De acordo com Andrade, Fabricante e Oliveira (2010), valores próximos de zero negativo representam sérios problemas ambientais para as comunidades autóctones.

A presença da Leucaena leucocephala pode ser explicada pela proximidade da mineração à rodovia SP 104/79 — João Guimarães, onde se observa a dominância da espécie. Sabendo que sua plasticidade ecológica permite adaptar-se a diferentes solos com baixa disponibilidade de nutrientes, a espécie foi capaz de se dispersar e colonizar as áreas degradadas da mineração.

Outro fator preponderante para os altos índices da Leucaena leucocephala se dá pela ausência de competidores e predadores que possam interromper seu estabelecimento e a ocorrência de seu ciclo de vida. Para Bourscheid e Reis (2010), muitas das espécies exóticas invasoras apresentam vantagens competitivas com relação às nativas por fazerem menos interações com a comunidade local. Ou seja, organismos introduzidos espalham-se rapidamente no novo ambiente, porque neste não existem os predadores, patógenos e herbívoros, com os quais coevoluíram em seu habitat de origem.

Verifica-se que, apesar de exótica, a Leucaena leucocephala passou a compor o ambiente em restauração, favorecendo, assim, a acumulação de nitrogênio. De acordo com Sanginga (1992), a Leucaena leucocephala forma simbiose eficiente com estirpes específicas de Rhizobium, podendo fixar de 200 a $300 \mathrm{~kg} \mathrm{ha}^{-1}$ de N, tornando esse elemento disponível em agrossistemas tropicais. De acordo com Chada, Campello e Faria (2004), as leguminosas são fartamente relatadas como recolonizadoras espontâneas de áreas degradadas pela mineração e como tendo grande sucesso em projetos de revegetação. Gonçalves et al. (2004) comentam que rusticidade, baixas exigências nutricionais e capacidade de nodular e fixar nitrogênio são consideradas as principais características que proporcionam às plantas dessa família maior capacidade de sobreviver em ambientes minerados.

Ao avaliar a influência da riqueza de Leucaena leucocephala sobre outras espécies (Figura 1), verifica-se que maior densidade aparentemente proporciona facilitação inicial para que outras espécies ocorram no local, relação que pode ser alterada de acordo com o tempo. Inicialmente na área I, a espécie atua como facilitadora no avanço da sucessão ecológica com 57,9\% em relação a 82,3\% na área II. Age ainda como abrigo para os vetores de dispersão, melhora as condições de fertilidade do solo e fornece habitáts adequados ao recrutamento, facilitando, dessa maneira, o estabelecimento de outras espécies. No entanto, ao longo do tempo, a espécie age como inibidora, dificultando a regeneração de plantas nativas nas pilhas de estéril (área II).

De acordo com Connell e Slatyer (1977), existem três modelos diferentes de sucessão. O primeiro é denominado de facilitação, no qual espécies secundárias só se estabelecem no local após as primárias terem modificado as condições ambientais; o segundo é a tolerância, no qual espécies secundárias não precisam necessariamente da presença de primárias para se estabelecerem e crescerem, pois o estabelecimento depende do nível individual de tolerância a escassez de recursos das espécies; e o terceiro é o de inibição, no qual as espécies primárias inibem o crescimento de outras por meio do uso de espaço e recursos.

Quanto à diversidade para a regeneração natural, foi observado que a abundância da Leucaena leucocephala interferiu negativamente no processo de sucessão ecológica, impedindo o estabelecimento das demais espécies. De acordo com Ziller e Zalba (2007), espécies invasoras competem severamente com as nativas pelos recursos naturais do meio, diminuindo o aporte de novos propágulos. Esse comportamento é típico de espécies ruderais agressivas.

Yoshida e Oka (2004) constataram que a recuperação da biodiversidade e da estrutura de florestas nativas é muito mais lenta em áreas ocupadas por Leucaena leucocephala do que em áreas não ocupadas por ela. A inibição causada por essa espécie pode ser atribuída à liberação

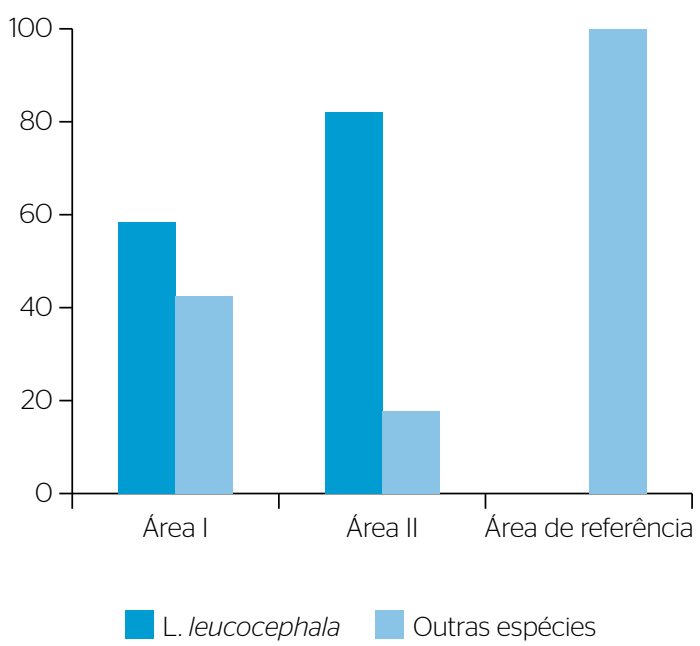

Figura 1 - Proporção da riqueza da Leucaena leucocephala nos diferentes ambientes em relação às demais espécies. 
de substâncias alopáticas. Segundo Chou e Kuo (1986), a fitotoxicidade do extrato de Leucaena leucocephala sobre várias plantas tem sido atribuída à diversidade de aleloquímicos presentes em sua composição: a mimosina (b-[N-(3-hidroxi-4-oxopiridil)]-a-aminopropiônico), a quercetina e os ácidos gálico, protecatequico, p-hidroxibenzoico, phidroxifenilacético, vanílico, ferúlico, caféico e p-cumárico. Esses dados corroboram os estudos conduzidos por Prates et al. (2000), os quais revelaram que o uso da parte aérea da Leucaena leucocephala - tanto em cobertura, como incorporada ao solo - reduziu a população de plantas daninhas presentes na cultura do milho, com maior efeito sobre as plantas de folhas largas.

Cabe destacar que, embora a utilização de espécies exóticas deva ser evitada em determinadas situações, no caso de solos expostos e sujeitos à erosão, tem sido recomendada por alguns autores em função de promover cobertura rápida e melhoria da fertilidade do solo, o que é particularmente importante no caso da mineração, além de possibilitar a formação de florestas catalisadoras, que facilitam a regeneração de espécies nativas no sub-bosque (PARROTTA; KNOWLES, 1999; SANTILLI; DURIGAN, 2014). Nesse sentido, Santilli e Durigan (2014) observaram que, mesmo em uma comunidade em restauração em que 94\% das árvores plantadas foram exóticas, após 8 anos, apenas 3\% das plantas regenerantes pertenciam a espécies exóticas, o que indica uma tendência de aumento de espécies nativas ao longo do tempo. Além do mais, Chavez (1994) aponta a espécie Leucaena leucocephala como uma das leguminosas que possuem usos múltiplos em sistemas agrissilvipastoris, sendo usada como suplementação alimentar para o gado, principalmente na época seca. Bertalot (1997) sugere o uso da Leucaena leucocephala como uma das leguminosas arbóreas fixadoras de nitrogênio com potencial para ecossistemas agroflorestais em solos de cerrado.

Nota-se ainda, na Figura 1, que, apesar de a Leucaena leucocephala estar presente nas pilhas de estéril, houve nenhuma ocorrência desta na AR. Observa-se que a espécie não invadiu o fragmento florestal nativo, uma vez que nenhum indivíduo foi registrado entre os 613 amostrados, e não afetou a sucessão dos ecossistemas florestais do local. Estudo realizado por Costa e Durigan (2010) aponta que a Leucaena leucocephala não se comportou como espécie invasora, uma vez que não se expandiu sobre ecossistemas naturais. A espécie seria, portanto, mais bem identificada como ruderal, uma vez que pode proliferar em áreas perturbadas e dificultar o estabelecimento de espécies nativas (WOITKE; DIETZ, 2002).

Ainda que a espécie Leucaena leucocephala reúna alguns predicados favoráveis à invasão, tais como porte arbóreo, crescimento rápido e grande produção de sementes, de modo que seja inibido o estabelecimento de outras espécies, seus indivíduos não atingem grande porte (LORENZI; TORRES, 2003). Sendo uma espécie pioneira heliófita, espera-se que a Leucaena leucocephala perca espaço na comunidade à medida que as árvores nativas sombreiem suas copas, dificultando, desse modo, sua reprodução e regeneração. Em experimento visando determinar os mecanismos que conferem resistência de comunidades vegetais à invasão, Green, Lake e O'Dowd (2004) constataram 100\% de mortalidade das plântulas de Leucaena leucocephala no interior da floresta intacta. Esses autores consideraram a baixa luminosidade como o mais importante filtro abiótico impedindo a invasão por essa espécie.

\section{CONCLUSÕES}

Foi constatada a ocorrência da regeneração natural nas pilhas de estéril com o aumento do incremento de indivíduos e espécies ao longo do tempo. A composição e estrutura fitossociológica indicaram que a vegetação presente nessas pilhas está em transição de floresta pioneira para um estágio sucessional mais avançado. Contudo, a Leucaena leucocephala estabeleceu-se mais expressivamente que as demais espécies, o que sugere que esta esteja inibindo o desenvolvimento de outras nessas pilhas. No entanto, não invadiu o fragmento florestal nativo.

Recomenda-se, então, o manejo da Leucaena leucocephala, assim como o enriquecimento com espécies florestais nativas, a fim de aumentar a diversidade do local para que a sucessão ecológica das áreas não seja comprometida.

\section{REFERÊNCIAS}

ANDRADE, L.A.; FABRICANTE, J.R.; OLIVEIRA, F.X. (2010) Impactos da invasão de Prosopis juliflora (Sw.) DC. (Fabaceae) sobre o estrato arbustivo-arbóreo em áreas de Caatinga no Estado da Paraíba, Brasil. Acta Scientiarum. Biological Sciences, Maringá, v. 32, n. 3, p. 249-255. https://doi.org/10.4025/actascibiolsci.v32i3.4535

ANGELO, J.C.M.; LENA, J.C.; DIAS, L.E.; SANTOS, J.B. (2002) Diversidade vegetal em áreas em reabilitação de mineração de ferro, na mina de Alegria, em Mariana, MG. Revista Arvore, Viçosa, v. 26, n. 2, p. 183-192.
ANGIOSPERM PHYLOGENY GROUP (APG III). (2009) An update of the Angiosperm Phylogeny Group classification for the orders and families of flowering plants: APG III. Botanical Journal of the Linnean Society, Londres, v. 161, n. 2, p. 105-121. https://doi.org/10.1111/ j.1095-8339.2009.00996.x

BERTALOT, M.J.A. (1997) Crescimento e avaliação nutricional de leguminosas arbóreas potenciais para ecossistemas agroflorestais num solo de cerrado. 63f. Dissertação (Mestrado em Agronomia) 
- Faculdade de Ciências Agronômicas, Universidade Estadual Paulista “Júlio de Mesquita Filho", Botucatu.

BOURSCHEID, K.; REIS, A. (2010) Dinâmica da invasão de Pinus elliottii Engelm. em restinga sob processo de restauração ambiental no Parque Florestal do Rio Vermelho, Florianópolis, SC. Biotemas, Florianópolis, v. 23, n. 2, p. 23-30. https://doi.org/10.5007/2175-7925.2010v23n2p23

BRANCALION, P.H.S.; GANDOLFI, S.; RODRIGUES, R.R. (2009) Incorporação do conceito da diversidade genética na restauração ecológica. In: RODRIGUES, R.R.; BRANCALION, P.H.S.; ISERNHAGEN, I. (orgs.). Pacto pela restauração da mata atlântica: referencial dos conceitos e ações de restauração florestal. São Paulo: LERF/ESALQ. p. 37-54.

BRASIL. (1989) Decreto no 97.632 - 10 de abril de 1989. Dispõe sobre a regulamentação do artigo 20, inciso VIII, da Lei no 6.938 de 31 de agosto de 1981, e dá outras providências. Diário Oficial da República Federativa do Brasil, Brasília, 10 abr. 1989. Disponível em: <http://www.planalto.gov.br/ccivil_O3/decreto/1980-1989/D97632. htm>. Acesso: 20 jul. 2016.

(2017) Decreto no 8.972, de 23 de janeiro de 2017. Institui a Política Nacional de Recuperação da Vegetação Nativa. Diário Oficial da República Federativa do Brasil, Brasília, 23 jan. 2017. Disponível em: <http://www.planalto.gov.br/ccivil_03/_Ato20152018/2017/Decreto/D8972.htm>. Acesso: 25 jan. 2017.

CARDOSO-LEITE, E.C.; RODRIGUES, R.R. (2008) Fitossociologia e caracterização sucessional de um fragmento de Floresta Estacional no sudeste do Brasil. Revista Árvore, Viçosa, v. 32, n. 3, p. 583-595. http://dx.doi.org/10.1590/S0100-67622008000300019

CENTRO DE PESQUISAS METEOROLÓGICAS E CLIMÁTICAS APLICADAS A AGRICULTURA (CEPAGRI); UNIVERSIDADE ESTADUAL DE CAMPINAS (UNICAMP). Clima dos Municípios Paulistas. Disponível em: <http://www.cpa.unicamp.br/outrasinformacoes/clima-dos-municipios-paulistas.htmls. Acesso em: 15 mar. 2016

CHADA, S.S.; CAMPELLO, E.F.C.; FARIA, S.M. (2004) Sucessão vegetal em uma encosta reflorestada com leguminosas arbóreas em Angra dos Reis, RJ. Revista Árvore, Viçosa, v. 28, n. 6, p. 801-809. http://dx.doi.org/10.1590/S0100-67622004000600005

CHAVEZ, S.V. (1994) Contenido de taninos y digestibilidad in vitro de algunos forrajes tropicales. Agroforestería en las Américas, Costa Rica, v. 1, n. 3, p. 10-13.

CHOU, C.H.; KUO, Y.L. (1986) Allelopathic research of subtropical vegetation in Taiwan: III. Allelopathic exclusion of understory by Leucaena leucocephala (Lam.) de Wit. Journal of Chemical Ecology, Washington, D.C., v. 12, p. 1431-1448. http://doi.org/10.1007/BF01012362

CIELO-FILHO, R.; SOUZA, J.A.D. (2016) Avaliação da restauração passiva de uma área de mata atlântica após o corte raso de uma plantação de Cupressus Iusitanica MILL. Ciência Florestal, Santa Maria, v.26, n.2, p. 475-488. http://dx.doi.org/10.5902/1980509822748

COELHO, S. (2013) Estudo da vegetação do Parque Natural Municipal Corredores da biodiversidade (PNMCBio), SOROCABA/ $S P$. 87f. Dissertação (Mestrado em Sustentabilidade na Gestão Ambiental) - Universidade Federal de São Carlos, Sorocaba.
CONNELL, J.; SLATYER, R. (1977) Mechanisms of succession in natural communities and their role in community stability and organization. American Naturalist, Chicago, v. 111, n. 982, p. 1119-1141.

CORREA, L.; CARDOSO-LEITE, E.; CASTELLO, A.; COELHO, S.; KORTZ, A.R.; VILLELA, F.N.J.; KOCH, I. (2O14) Estrutura, composição florística e caracterização sucessional em remanescente de Floresta Estacional Semidecidual no sudeste do Brasil. Revista Árvore, v. 38, n. 5, p. 799-809. http://dx.doi.org/10.1590/S010067622014000500004

COSTA, J.N.M.N. da; DURIGAN, G. (2010) Leucaena leucocephala (Lam.) de Wit (Fabaceae): Invasora ou ruderal? Revista Árvore, Viçosa, v. 34, n. 5, p. 825-833. http://dx.doi.org/10.1590/S010067622010000500008

DISLICH, R.; CERSÓSIMO, L.; MANTOVANI, W. (2001) Análise da estrutura de fragmentos florestais no Planalto Paulistano-SP. Revista Brasileira de Botânica, São Paulo, v. 24, n. 3, p. 321-332. http:// dx.doi.org/10.1590/S0100-84042001000300011

FLORA DO BRASIL 202O. Jardim Botânico do Rio de Janeiro. Disponíve em: <http://floradobrasil.jbrj.gov.br/>. Acesso em: 10 dez. 2016.

GANDOLFI, S.; LEITÃO FILHO, H.F.; BEZERRA, C.L.F. (1995) Levantamento florístico e caráter sucessional das espécies arbustivo-arbóreas de uma floresta semidecídua no município de Guarulhos, SP. Revista Brasileira de Biologia, Rio de Janeiro, v. 55, n. 4, p. 753-767.

GONÇALVES, J.L.M. SANTARELLI, E.G.; MORAES NETTO, S.P.; MANARA, M.P. (2004) Seedling production of native species: substrate, nutrition, shading, and fertilization. In: GONÇALVES, J.L.M.; BENEDETTI, V. (orgs.). Forest nutrition and fertilization. Piracicaba: Instituto de Pesquisas Florestais e Estudos Florestais. p. 307-345.

GREEN, P.T.; LAKE, P.S.; O'DOWD, D.J. (2004) Resistance of Island Rainforest to Invasion by Alien Plants: Influence of Microhabitat and Herbivory on Seedling Performance. Biological Invasions, Tennessee, v. 6, n. 1, p. 1-9. https://doi.org/10.1023/ B:BINV.0000010144.12808.cb

HOLL, K.D. (2002) Effect of shrubs on tree seedling establishment in an abandoned tropical pasture. Journal of Ecology, Londres, v. 90, n. 1, p. 179-187.

INSTITUTO AMBIENTAL DO PARANÁ (IAP). (2009) Portaria IAP no 125, de 7 de agosto de 2009. Reconhece a Lista Oficial de Espécies Exóticas Invasoras para o Estado do Paraná, estabelece normas de controle e dá outras providências. Disponível em: <http://www.iap. pr.gov.br/arquivos/File/Lista_invasoras_PR_corrigida_set_2015.pdf>. Acesso em: 13 jan. 2017.

(2015) Lista de Espécies Exóticas Invasoras é atualizada no Paraná. Disponível em: <http://www.iap.pr.gov.br/modules/noticias/ article.php?storyid=705/ >. Acesso em: 13 jan. 2017.

INSTITUTO BRASILEIRO DE GEOGRAFIA E ESTATISTTICA (IBGE) (2012) Manuais Técnicos em Geociências: Manual Técnico da Vegetação Brasileira. n. 1. Rio de Janeiro: IBGE. Disponível em: <http://biblioteca.ibge.gov.br/visualizacao/livros/liv63011.pdf> Acesso em: 20 jun. 2016. 
LORENZI,H.; TORRES, H.M.(2003)Árvores Exóticas no Brasil:madeireiras, ornamentais e aromáticas. Nova Odessa: Instituto Plantarum.

MAGNAGO, L.F.S.; MARTINS, S.V.; VENZKE, T.S.; IVANAUSKAS, N.M. (2012) Os processos e estágios sucessionais da mata atlântica como referência para a restauração florestal. In: MARTINS, S.V. (org.). Restauração ecológica de ecossistemas degradados. Viçosa: Editora da UFV. p. 69-100.

MAGURRAN, A.E. (1988) Ecological Diversity and its measurement. Nova Jersey: Princeton University Press.

MARTINS, S.V.; COUTINHO, M.P.; MARANGON, L.C. (2002) Composição florística e estrutura de uma floresta secundária no município de Cruzeiro, SP. Revista Árvore, Viçosa, v. 26, n. 1, p. 35-41.

MCINTIRE, E.J.B.; FAJARDO, A. (2014) Facilitation as a ubiquitous driver of biodiversity. New Phytologist, Lancaster, v. 201, n. 2, p. 403-416.

MIRANDA NETO, A.; MARTINS, S.V;; SILVA, K.A.; GLERIANI, J.M. (2012) Estrato de regeneração natural de uma floresta restaurada com 40 anos. Pesquisa Florestal Brasileira, Colombo, v. 32, n. 72, p. 409-420. https://doi.org/10.4336/2012.pfb.32.72.409

MUELLER-DOMBOIS, D.; ELLENBERG, H. (1974) Aims and methods of vegetation ecology. Nova York: John Wiley \& Sons.

PARROTTA, J.A.; KNOWLES, O.H. (1999) Restoration of tropical moist forest on bauxite mined lands in the Brazilian Amazon. Restoration Ecology, Washington, D.C., v. 7. n. 2, p. 103-116. https:// doi.org/10.1046/j.1526-100X.1999.72001.x

PARROTTA, J.A.; KNOWLES, O.H.; WUNDERLE JR., J.M. (1997) Development of floristic diversity in 10 -yearold restoration Forest on a bauxite mined site in Amazônia. Forest Ecology and Management, Berkeley, v. 99, n. 1-2, p. 21-42. https://doi.org/10.1016/ SO378-1127(97)00192-8

PASSINI, T.; KRANZ, W.M. (1997) Eficácia de herbicidas no controle de amarelinho (Tecoma stans) em pastagem. Planta Daninha, v. 15, n. 2, p. 190-197.

PRACH, K.; WALKER, L.R. (2011) Four opportunities for studies of ecological succession. Trends in Ecology and Evolution, Londres, v. 26, n. 3, p. 119-123. https://doi.org/10.1016/j.tree.2010.12.007

PRATES, H.T.; PAES, J.M.V; PIRES, N.M.; PEREIRA FILHO, I.A.; MAGALHÃES, P.C. (2000) Efeito do extrato aquoso de leucena na germinação e no desenvolvimento do milho. Pesquisa Agropecuária Brasileira, Brasília, v. 35, n. 5, p. 909-914. http://dx.doi. org/10.1590/S0100-204X2000000500007

REASER, J.K.; MEYERSON, L.A.; CRONK, Q; POORTER, M.; ELDREGE, L.G.; GREEN, E.; KAIRO, M.; LATASI, P.; MACK, R.N.; MAUREMOOTOO, J.; O'DOWD, D.; ORAPA, W.; SASTROUTOMO, S.; SAUNDERS, A.; SHINE, C.; THRAINSSON, S.; VAIUTU, L. (2007) Ecological and socioeconomic impacts of invasive alien species in island ecosystems. Environmental Conservation, Rhode Island, v. 34, n. 2, p. 98-111. https://doi.org/10.1017/S0376892907003815
RODRIGUES, E.R.; MONTEIRO, R.; CULLEN, L.J.R. (2010) Dinâmica inicial da composição florística de uma área restaurada na região do Pontal do Paranapanema, São Paulo, Brasil. Revista Árvore, Viçosa, v. 34 n. 5, p. 853-861. http://dx.doi.org/10.1590/S0100-67622010000500010

SAMPAIO, J.A.; ALMEIDA, S.L.M. (2005) Calcário e Dolomito. In: LUZ, A.B.; LINS, F.A.F. Rochas e Minerais Industriais. 2. ed. Rio de Janeiro: CETEM/MCT.

SANGINGA, N. (1992) Nitrogen fixation by trees and its contribution to the nitrogen status of soils or associated crops. In: HAWKINS, A.P.; CRAWFORD, K.M. (orgs.). Interactions between plants and microorganisms. Dakar: Fondation Internationale pour la Science. p. 14-32.

SANTILLI, C.; DURIGAN, G. (2014) Do alien species dominate plant communities undergoing restoration? A case study in the Brazilian savanna. Scientia Forestalis, Piracicaba, v. 42, n. 103, p. 371-382.

SARTORI, M.S. (2001) Variação da regeneração natural da vegetação arbórea no sub-bosque de Eucalyptus saligna Smith, manejado por talhadia, localizado no município de Itatinga, SP. 84f. Dissertação (Mestrado em Ciências Florestais) - Escola Superior de Agricultura Luiz de Queiroz, Universidade de São Paulo, Piracicaba.

SHEPHERD, G.J. (2009) FITOPAC 2.1 (versão preliminar). Campinas: Departamento de Biologia Vegetal, Universidade Estadual de Campinas.

SILVA, A.P.M.; VIANA, J.P.; CAVALCANTE, A.L.B. (2011) Resíduos Sólidos da Atividade de Mineração. Caderno de Diagnóstico. 41 p.

SOUZA, V.C.; LORENZI, H. (2008) Botânica sistemática: guia ilustrado para identificação das famílias de fanerógamas nativas e exóticas no Brasil, baseado em APG II. 2. ed. Nova Odessa: Instituto Plantarum.

TABARELLI, M. (1994) Clareiras Naturais e a Dinâmica Sucessional de um Trecho de Floresta na Serra da Cantareira, SP. 142f. Dissertação (Mestrado em Ecologia) - Instituto de Biociências, Universidade de São Paulo, São Paulo.

WOITKE, M.E.; DIETZ, H. (2002) Shifts in dominance of native and invasive plants in experimental patches of vegetations. Perspectives in Plant Ecology, Evolution and Systematics, Würzburg, v. 5, n. 3, p. 165-184. https://doi.org/10.1078/1433-8319-00032

WOLDA, H. (1981) Similarity indices, sample size and diversity. Oecologia, Nova York, v. 50, n. 3, p. 296-302. Disponível em: <https:// deploy.extras.ufg.br/projetos/adrimelo/div/Wolda1981.pdf>. Acesso em: 7 out. 2016.

YOSHIDA, K.; OKA, S. (2004) Invasion of Leucaena leucocephala and its effects on the native plant community in the Ogasawara (Bonin) Islands. Weed Technology, Lawrence, v. 18, p. 1371-1375. https://doi.org/10.1614/0890-037X(2004)018[1371:IOLLAI]2.0.CO;2

ZILLER, S.R.; ZALBA, S. (2007) Propostas de ação para prevenção e controle de espécies exóticas invasoras. Natureza e Conservação, Florianópolis, v. 5, n. 2, p. 8-15. 